Research Article

\title{
On Some Structural Components of Nilsolitons
}

\author{
Hulya Kadioglu \\ Department of Mathematics Education, Yildiz Technical University, Esenler 34210, Istanbul, Turkey
}

Correspondence should be addressed to Hulya Kadioglu; kadiogluhulya@gmail.com

Received 17 February 2021; Accepted 7 April 2021; Published 17 May 2021

Academic Editor: Mustafa Inc

Copyright (c) 2021 Hulya Kadioglu. This is an open access article distributed under the Creative Commons Attribution License, which permits unrestricted use, distribution, and reproduction in any medium, provided the original work is properly cited.

In this paper, we study nilpotent Lie algebras that admit nilsoliton metric with simple pre-Einstein derivation. Given a Lie algebra $\eta$, we would like to compute as much of its structure as possible. The structural components we consider in this study are the structure constants, the index, and the rank of the nilsoliton derivations. For this purpose, we prove necessary or sufficient conditions for an algebra to admit such metrics. Particularly, we prove theorems for the computation of the Jacobi identity for a given algebra so that we can solve the system of the equation(s) and find the structure constants of the nilsoliton.

\section{Introduction}

On any Lie group, it is possible to define several different Riemannian metrics. Considering any Riemannian metrics, Einstein metrics are the most preferable metrics, as the Ricci tensor complies the Einstein metric: Ric $=\mathrm{cg}$, for some constant $c \in \mathbb{R}$. But, it is not possible to define Einstein metrics on nonabelian nilpotent Lie algebras; therefore, we consider the following weaker condition on a left invariant metric $g$ on a nilpotent Lie group $G$ :

$$
\operatorname{Ric}_{g}=\beta I+D \text {, }
$$

for some $\beta \in \mathbb{R}$ and $D \in \operatorname{Der}(\eta)$, where $\operatorname{Ric}_{g}$ denotes the Ricci operator of $(\eta, g), \eta$ is the Lie algebra of $G$, and $\operatorname{Der}(\eta)$ denotes the Lie algebra of derivations of $\eta$. Equation (1) is called the nilsoliton condition, $D$ is called the nilsoliton derivation, and $\beta$ is called the nilsoliton constant.

Nilsoliton metric Lie algebras are unique up to isometry and scaling. This is one of the reasons that makes nilsolitons an important topic. On the other hand, there is a one to one correspondence between Einstein nilradicals with nilsolitons. In [1], Theorem 2.11 states that a nilpotent Lie algebra $\eta$ is an Einstein nilradical if and only if $\eta$ admits a nilsoliton metric. Therefore, it indicates that classification of nilsoliton metrics on a nilpotent Lie algebra is equivalent to the same of Einstein nilradicals. Additionally, if $\delta$ is Einstein solvmanifold, then the metric restricted to the submanifold can completely be determined by the nilsoliton metric Lie algebra $\eta=[\delta, \delta]$. On the contrary, any nilsoliton can uniquely extend to an Einstein solvmanifold. Therefore, the study of solvmanifolds is actually the study of nilsolitons. See $[1,2]$, for a survey on nilsoliton metric Lie algebras.

There are three methods to represent a Lie algebra and its related structures: representing a Lie algebra as a linear Lie algebra, i.e., subalgebra of $\mathrm{gl}(n)$, using table of its structure constants or using generators and relations [3]. In this paper, we use the table of structure constants related to a Lie algebra. The main reason is that this representation helps one to create and classify Lie algebras by the computer software programs as in [4-6]. We vary the Lie algebra structure by finding structure constants. Namely, we determine a Lie algebra $\eta$ with a fixed basis $\left\{X_{i}: 1 \leq i \leq n\right\}$ explicitly by given multiplication table, consisting of structure constants $\alpha_{i j}^{k}$ which are defined by the relations

$$
\left[X_{i}, X_{j}\right]=\sum \alpha_{i j}^{k} X_{k} \text {. }
$$

In this work, the nonzero structure constants are encoded by using the index set $\Lambda=\left\{(i, j, k) \mid \alpha_{i j}^{k} \neq 0, i<j<k\right\}$, ignoring repetitions due to skew-symmetry. While indexing the structure constants, we use triples $(i, j, k) \in \Lambda$ such that $i<j<k$, and if $(i, j, k),(i, j, m) \in \Lambda$, then $k=m$ and $\left(i, j_{1}, k\right),\left(i, j_{2}, k\right) \in \Lambda$, then $j_{1}=j_{2}$. For this purpose, we fix a basis $\left\{X_{1}, \ldots, X_{n}\right\}$ for a nilpotent Lie algebra $\eta$ with $[X i, X j]=\sum \alpha_{i j}^{k} X_{k} \neq 0 \quad$ such that for every 
$i, j, \#\left\{k: \alpha_{i j}^{k} \neq 0\right\} \leq 1$, and for every $i, k, \#\left\{j: \alpha_{i j}^{k} \neq 0\right\} \leq 1$. Such basis $\left\{X_{j}\right\}$ is called nice and defined by Nikolayevsky in [7]. In our paper, we call this basis as "Nikolayevsky basis." Using this special basis, we do not need to use sum symbol in equation (2).

In this paper, we prove theorems regarding to some structural components of nilsolitons for possible algorithmic approach in classifications. In particular, we present some new concepts and theorems regarding to create Jacobi identity equations for a given nilsoliton metric Lie algebra. We also present methods for the computations of the index and the rank of a nilsoliton. We provide several examples to illustrate the newly proposed concepts and methods.

This paper consists of four sections. In the second section, we present preliminary background. In the third section, we prove necessary theorems that help us to calculate structural elements of nilsolitons. In the last section, we present concluding statements.

\section{Preliminaries}

Let $\left(\eta_{\mu},<,>\right)$ be a metric algebra, where $\mu \in \Lambda^{2} \eta \otimes \eta^{*}$. Let $B=\left\{X_{i}\right\}_{i=1}^{n}$ be a $\langle$,$\rangle -orthonormal basis of \eta_{\mu}$ (we always assume that basis is ordered). The nil-Ricci endomorphism $\operatorname{Ric}_{\mu}$ is defined as $\left.\left\langle\operatorname{Ric}_{\mu} X, Y\right\rangle\right\rangle=\operatorname{ric}_{\mu}(X, Y)$, where

$$
\begin{aligned}
\operatorname{ric}_{\mu}(X, Y)= & -\frac{1}{2} \sum_{i=1}^{n}\left\langle\left[X, X_{i}\right],\left[Y, X_{i}\right]\right\rangle \\
& +\frac{1}{4} \sum_{i=1}^{n}\left\langle\left[X_{i}, X_{j}\right], X\right\rangle\left\langle\left[X_{i}, X_{j}\right], Y\right\rangle,
\end{aligned}
$$

for $X, Y \in \eta$. When $\eta$ is a nilpotent Lie algebra, the nil-Ricci endomorphism is the Ricci endomorphism. If all elements of the basis are eigenvectors for the nil-Ricci endomorphism $\mathrm{Ric}_{\mu}$, we call the orthonormal basis a Ricci eigenvector basis.

Let $\operatorname{Der}(\eta)$ denote the derivation algebra of $\eta$. A maximal abelian subalgebra of $\operatorname{Der}(\eta)$ comprised of semisimple elements which is called a maximal torus. The dimension of a maximal torus is called the rank of $\eta$.

In the following, we define root vectors and root matrix, Gram matrix, Nikolayevsky basis.

Definition 1. Suppose that $\Lambda=\left\{(i, j, k) \mid \alpha_{i j}^{k} \neq 0, i<j<k\right\}$ is a finite set which indexes the set of nonzero structure constants corresponding to a Lie algebra $\eta$, ignoring repetitions due to skew-symmetry. For $1 \leq i, j, k \leq n$, we define $1 \times n$ row vector $y_{i j}^{k}$ to be $\epsilon_{i}^{T}+\epsilon_{j}^{T}-\epsilon_{k}^{t}$, where $\{\epsilon\}_{i=1}^{n}$ is the standard orthonormal basis for $\mathbb{R}^{n}$. We call the vectors in $\left\{y_{i j}^{k} \mid(i, j, k) \in \Lambda\right\}$ root vectors for $\Lambda$. Let $y_{1}, y_{2}, \ldots, y_{m}$ (where $m=|\Lambda|$ ) be an enumeration of the root vectors in dictionary order. We define root matrix $Y_{\Lambda}$ for $\Lambda$ to be the $m \times n$ matrix whose rows are the root vectors $y_{1}, y_{2}, \ldots, y_{m}$.

The Gram matrix $U_{\Lambda}$ for $\Lambda$ is the $m \times m$ matrix defined by $U_{\Lambda}=Y_{\Lambda} Y_{\Lambda}^{T}$; the $(i, j)$ entry of $U_{\Lambda}$ is the inner product of the $i$ th and $j$ th root vectors (unless otherwise stated, the matrix $U$ means the Gram matrix corresponding to the index set $\Lambda$. Therefore, from now on, we do not use $U_{\Lambda}$ ). From Theorem 5, in [8], we know that $U$ is a symmetric matrix where its all diagonal entries are 3 and its off-diagonal entries are in the set $\{-2,-1,0,1,2\}$.

Nikolayevsky showed that every Lie algebra admitting a derivation with all the eigenvalues of multiplicity one has a nice basis [7]. We use this type of basis in our study. This way our Gram matrices corresponding to metric nilpotent Lie algebras does not have a 2 and -2 as an entree (Lemma 2 in [4]).

Now, suppose that $|\Lambda|=m$ and $[1]_{m}$ represents a column vector $\left[\begin{array}{lllll}1 & 1 & 1 & \ldots & 1\end{array}\right]^{T}$ in $\mathbb{R}^{m}$.

Theorem 1 (Theorem 1 in [8]). Let $\eta$ be a nonabelian metric algebra with Ricci eigenvector basis $B$. Let $U$ and $\left[\alpha^{2}\right]$ be the Gram matrix and the structure vector for $\eta$ with respect to $B$. Then, $\eta$ satisfies the nilsoliton condition with nilsoliton constant $\beta$ if and only if $U\left[\alpha^{2}\right]=2 \beta[1]_{m}$.

The above theorem indicates a Lie algebra $\eta$ which admits a nilsoliton metric iff there exists a solution $v \in \mathbb{R}^{m}$ of the linear system $U v=[1]_{m}$ where all entries are positive real numbers.

\section{The Structural Elements}

In this section, we introduce notions and prove theorems for the computation structural elements of the nilsoliton metric Lie algebras.

3.1. The Jacobi Identity(s). Now, we present the theorems which help to create possible Jacobi identity equations.

Theorem 2 (see [8]). Let $\eta$ be an $n$-dimensional vector space; $B=\left\{X_{i}\right\}_{i=1}^{n}$ be a basis for $\eta$. Suppose that a set of nonzero structure constants $\alpha_{i, j}^{k}$ relative to $B$, indexed by $\Lambda$, defines a skew symmetric product on $\eta$. Assume that if $(i, j, k) \in \Lambda$, then $i<j<k$. Then, $\eta$ is a Lie algebra if and only if whenever there exists $m$ so that the inner product of root vectors $\left\langle y_{i j}^{l}, y_{l k}^{m}\right\rangle=-1$ for triples $(i, j, l)$ and $(l, k, m)$ or $(k, l, m)$ in $\Lambda$, the equation

$$
\sum_{s<m} \alpha_{i, j}^{s} \alpha_{s, k}^{m}+\alpha_{j, k}^{s} \alpha_{s, i}^{m}+\alpha_{k, i}^{s} \alpha_{s, j}^{m}=0,
$$

holds. Furthermore, a term of form $\alpha_{i, j}^{l} \alpha_{l, k}^{m}$ is nonzero if and only if $\left\langle y_{i, j}^{l}, y_{l, k}^{m}\right\rangle=-1$.

Lemma 1 (Lemma 2.8 in [4]). Let $\eta$ be an n-dimensional nonabelian nilpotent Lie algebra. Suppose that $\eta$ admits a derivation $D$ having distinct real positive eigenvalues. Let $B$ be a basis consisting of eigenvectors for the derivation $D$, and let $\Lambda$ index the nonzero structure constants with respect to $B$. Let $Y$ be the $m \times n$ root matrix for $\Lambda$. If $\operatorname{rank}(Y)=m$, then the following hold:

(1) $|\Lambda| \leq n-1$.

(2) If $\left(i_{1}, j_{1}, k_{1}\right) \in \Lambda \quad$ and $\quad\left(i_{2}, j_{2}, k_{2}\right) \in \Lambda$, then
$\left\langle y_{i_{1} j_{1}}^{k_{1}}, y_{i_{2} j_{2}}\right\rangle \neq-1$.

Remark 1. The reverse of Lemma 1 is not true. 
Proof. Suppose that $\eta$ is an algebra which is indexed by the following index set:

$$
\Lambda=\{(1,2,5),(1,3,6),(1,5,7),(1,6,8),(2,4,7),(2,5,8),(3,4,8)\} .
$$

Its corresponding Gram matrix is as follows:

$$
\left(\begin{array}{lllllll}
3 & 1 & 0 & 1 & 1 & 0 & 0 \\
1 & 3 & 1 & 0 & 0 & 0 & 1 \\
0 & 1 & 3 & 1 & 1 & 1 & 0 \\
1 & 0 & 1 & 3 & 0 & 1 & 1 \\
1 & 0 & 1 & 0 & 3 & 1 & 1 \\
0 & 0 & 1 & 1 & 1 & 3 & 1 \\
0 & 1 & 0 & 1 & 1 & 1 & 3
\end{array}\right) .
$$

It is a singular matrix with nullity $=1$. Also, it does not have -1 entry. From Theorem 2, it is a Lie algebra. Also, since the solution space of $U . v=[1]$ is

$$
\left\{v=\left(t+\frac{1}{19}, \frac{6}{19}-t, t, \frac{5}{19}-t, \frac{5}{19}-t, \frac{3}{19}, t\right) \mid 0<t<\frac{5}{19}\right\}
$$

from [8] that it is a nilsoliton metric Lie algebra with the magnitudes of the structure constants

$$
\begin{aligned}
& \left|\alpha_{1,2}^{5}\right|=t+\frac{1}{19}, \\
& \left|\alpha_{1,3}^{6}\right|=\frac{6}{19}-t, \\
& \left|\alpha_{1,5}^{7}\right|=t, \\
& \left|\alpha_{1,6}^{8}\right|=\frac{5}{19}-t, \\
& \left|\alpha_{2,4}^{7}\right|=\frac{5}{19}-t, \\
& \left|\alpha_{2,5}^{8}\right|=\frac{3}{19}\left|\alpha_{3,4}^{8}\right|=t,
\end{aligned}
$$

with simple derivation of type $7<10<13<$ $14<17<20<24<27$ with singular Gram matrix $U$ and $|\Lambda|=n-1$.

The counter example provided in Remark 1 illustrates none of the inner products of root vectors being -1 does not imply that the Gram matrix of the nilsoliton is nonsingular. Additionally, if the cardinality of the index set $|\Lambda| \leq n,-1$ does not imply that the Gram matrix is nonsingular.

3.1.1. Constructing the System of Jacobi Identities. In order an algebra to be a Lie algebra, one needs to satisfy the Jacobi identity. Using our index set $\Lambda$, the corresponding Jacobi identity turns into equation (4). Also, there has to be at least two product couples in the Jacobi identity. Otherwise, if there is one product couple in Jacobi identity, it leads to $\alpha_{i, j}^{s} \alpha_{s, k}^{m}=0$; therefore, it contradicts to the fact that $(i, j, s),(s, k, m) \in \Lambda$. On the other hand, the Jacobi identity is created by each vector triples from the given fixed Nikolayevsky basis. For example, the Jacobi identity for $X_{i}, X_{j}, X_{k}$ is

$$
\alpha_{i j}^{s} \alpha_{s k}^{m}+\alpha_{j k}^{s} \alpha_{s i}^{m}+\alpha_{k i}^{s} \alpha_{s j}^{m}=0
$$

Since we use the Nikolayevsky basis, there is a unique $k$ which appears in equation (9). Therefore, each Jacobi identity is created with fixed $X_{i}, X_{j}, X_{k}$, and $m \in$ AoM. Also, because there has to be at least one product couple in the Jacobi identity, then there exist 2 or three product couples in the Jacobi identity. In equation (9), the product couple $\alpha_{i j}^{s} \alpha_{s k}^{m}$ corresponds to the index triples in $\Lambda$ such that $(i, j, s),(s, k, m) \in \Lambda$, or $(i, j, s),(k, s, m) \in \Lambda$. In the following definition, we define the set of all product couples $P_{m}$ related to the Jacobi identity for a given subset $X_{i}, X_{j}, X_{k}$ of the fixed basis. As one can see, there exist at most 3 product couples in the same Jacobi identity.

Definition 2. If there are $t$ product couples in the Jacobi identity for the same $m$ such that $(i, j, s),(s, k, m) \in \Lambda$, or $(i, j, s),(k, s, m) \in \Lambda$, then the Jacobi identity which was created by the basis vectors $\left\{X_{i}, X_{j}, X_{k}\right\}$. Now, we define the set of all nonzero product couples for a given index triple basis vectors $X_{i}, X_{j}, X_{k}$ as follows:

$$
P_{m}=\left\{p_{s}=\left|\alpha_{i j}^{s} \alpha_{s, k}^{m}\right|, 1 \leq s \leq n, p_{s} \neq 0\right\} .
$$

It is called set of product couples for $\# m$ and for in equation (4):

$$
\operatorname{AoM}=\{m \in \mathbb{N}:(i, j, s),(s, k, m) \in \Lambda \text { or }(i, j, s),(k, s, m) \in \Lambda\} .
$$
(4).

Here, AoM is the matrix of \#m that appears in equation

Remark 2. Let $\eta$ be an algebra that is indexed by $\Lambda$, then the Jacobi identity for a given $X_{i}, X_{j}, X_{k}$ with each $m \in$ AoM is given by

$$
p_{1} \mp p_{2} \mp p_{3}=0 \text {. }
$$

The proof of Remark 2 follows from the definition of Jacobi identity for a given algebra.

Theorem 3. Let $\eta$ be an algebra that is indexed by $\Lambda$, and $U$ be the Gram matrix related to $\Lambda$. Let $v_{p}$ belong to the solution space to the linear system $U v=[1]$, then the Jacobi identity for each $m \in A o M$ is given by

$$
\sqrt{v_{i_{1}} v_{j_{1}}} \mp \sqrt{v_{i_{2}} v_{j_{2}}} \mp \sqrt{v_{i_{3}} v_{j_{3}}}=0
$$

where $1 \leq i_{1}, i_{2}, i_{3}, j_{1}, j_{3} \leq|\Lambda|$.

Proof. Let $\eta$ be an algebra that is indexed by $\Lambda$. By Remark 3, the Jacobi identity for each $m \in$ AoM and each basis vector triple $X_{i}, X_{j}, X_{k}$ is given by 


$$
p_{1} \mp p_{2} \mp p_{3}=0 \text {. }
$$

Here, $p_{s}=\alpha_{i j}^{s} \alpha_{s, k}^{m}$. By Theorem 1, the solution vectors of the linear system $U v=[1]$ are the squares of the structure vectors $\alpha_{i j}^{s}$ for all $(i, j, s) \in \Lambda$. Therefore, for any $v$ that satisfies $U v=[1]$ is of form $v=\left(\alpha_{i, j}^{s}\right)^{2}$. Thus, for each $p_{s} \in P_{m}$,

$$
p_{s}=\sqrt{v_{a} v_{b}}
$$

where $a, b \in\left\{i_{1}, i_{2}, i_{3}, j_{1}, j_{3}\right\}$, and $v_{a}=\left(\alpha_{i j}^{s}\right)^{2}$ and $v_{b}=\left(\alpha_{s k}^{m}\right)^{2}$. Solving the equations for the structure constants, we have

$$
\alpha_{i j}^{s}=\mp \sqrt{v_{a}},
$$

for all $(i, j, s) \in \Lambda$. Therefore, equation (12) turns into

$$
\sqrt{v_{i_{1}} v_{j_{1}}} \mp \sqrt{v_{i_{2}} v_{j_{2}}} \mp \sqrt{v_{i_{3}} v_{j_{3}}}=0 \text {, }
$$

which finishes the proof.

In some cases, there can be more than two square root product couples in equation (13), i.e., there is more than one $m \in$ AoM. In that case, we need to consider all the cases of the signs between the product couples $\alpha_{i, j}^{s} \alpha_{s, k}^{m}, \alpha_{j, k}^{s} \alpha_{s, i}^{m}$, and $\alpha_{k, i}^{s} \alpha_{s, j}^{m}$. The following lemma deals with this matter.

Lemma 2. If there are $k$ product couples in the Jacobi identity, then there are $2^{k-1}-1$ possible sign choices.

Proof. Suppose that $p_{i} \in P_{m}$ for $m$ in equation (4) where $P_{m}$ is the set of all product couples, as in Definition 2. Therefore, the Jacobi identity turns into $p_{1} \mp p_{2} \mp p_{3}=0$. Without loss of generality, we assume that $p_{1}>0$. For each $p_{s} \in P_{m}$, where $2 \leq s \leq n$, there are two possible sign choices $\{+,-\}$. Therefore, we have $2^{t-1}$ possible sign choices. Since all the product couples are nonzero, then they can not all be + . Therefore, we drop the case $(+,+, \ldots,+)$. Thus, there are $2^{t-1}-1$ possible sign choices for the set $P_{m}$.
As we know that there is at most three, at least two product couples in the Jacobi identity, the there is at most three different sign choices for a Jacobi identity.

Definition 3. We define the matrix of all sign choices $\mathrm{SC}=$ $\left[s_{j s}\right] \in M_{\left(2^{t-1}-1\right) \times(t-1)}$ matrix where the entries are of the form

$$
s_{j s}= \begin{cases}1, & p_{s}>0, \\ 0, & p_{s}<0,\end{cases}
$$

for $1 \leq j \leq 2^{t-1}-1$. For example, if $P_{m}=\left\{p_{1}, p_{2}, p_{3}\right\}$, then $S C$ is a $3 \times 2$ matrix

$$
S C=\left(\begin{array}{ll}
1 & 0 \\
0 & 1 \\
0 & 0
\end{array}\right) .
$$

The equations are created by

$$
\begin{aligned}
& p_{1}+(-1)^{S C(1,1)} \cdot p_{2}+(-1)^{S C(1,2)} \cdot p_{3}=0 \\
& p_{1}+(-1)^{S C(2,1)} \cdot p_{2}+(-1)^{S C(2,2)} \cdot p_{3}=0 \\
& p_{1}+(-1)^{S C(3,1)} \cdot p_{2}+(-1)^{S C(3,2)} \cdot p_{3}=0
\end{aligned}
$$

Therefore, we have $p_{1}+p_{2}-p_{3}=0, p_{1}-p_{2}+p_{3}=0$, and $p_{1}-p_{2}-p_{3}=0$. If there is more than one array of number $m$ 's in equation (4) in Theorem 2 , then one needs to find common solutions of at least one Jacobi identity that was created by SC matrix for each $m$. To illustrate the above procedure, we have the following example.

\section{Example 1. Suppose that}

$$
\begin{aligned}
\Lambda=\{(1,3,4),(1,4,5),(1,5,6),(2,3,6),(1,6,7), \\
\\
(2,4,7),(1,7,8),(2,5,8)\} .
\end{aligned}
$$

The solution of the equation $U v=[1]$ is of the form

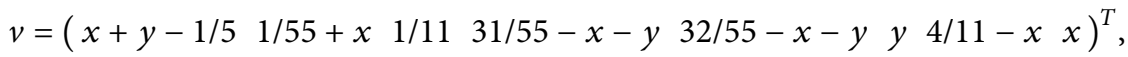

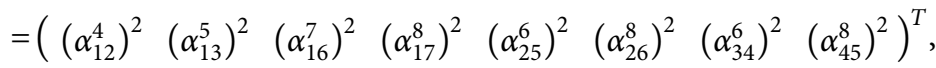

where $x, y$ are parameters of the solution system. Then, there are two $m$ 's in equation (4). Now, let us denote the set of $m$ as AoM. Therefore, AoM $=\{7,8\}$. Each $m \in$ AoM corresponds to a Jacobi identity. For $m=7$, we have two product couples of the triples $(i, j, l)$ and $(l, k, m)$ or $(k, l, m)$ in $\Lambda$. They are $(2,3,6)(1,6,7)$ and $(1,3,4)(2,4,7)$. Therefore, the first Jacobi identity is

$$
\left(\alpha_{23}^{6}\right)\left(\alpha_{16}^{7}\right) \pm\left(\alpha_{13}^{4}\right)\left(\alpha_{24}^{7}\right)=0
$$

For $m=8$, we have two product couples of the triples $(i, j, l)$ and $(l, k, m)$ or $(k, l, m)$ in $\Lambda$. They are $(2,4,7)(1,7,8)$ and $(1,4,5)(2,5,8)$. So, the corresponding Jacobi identity is

$$
\left(\alpha_{24}^{7}\right)\left(\alpha_{17}^{8}\right) \pm\left(\alpha_{14}^{5}\right)\left(\alpha_{25}^{8}\right)=0
$$

It can easily be seen that there is a unique sign choice for the above equations. The common solution is as follows: 


$$
\begin{aligned}
& \alpha_{13}^{4}=\sqrt{2 / 11} \\
& \alpha_{14}^{5}=\sqrt{1 / 5} \\
& \alpha_{15}^{6}=\sqrt{1 / 11} \\
& \alpha_{23}^{6}=\sqrt{2 / 5} \\
& \alpha_{16}^{7}=\sqrt{1 / 5} \\
& \alpha_{24}^{7}=\sqrt{1 / 5} \\
& \alpha_{17}^{8}=\sqrt{2 / 11} \\
& \alpha_{25}^{8}=\sqrt{2 / 11}
\end{aligned}
$$

3.2. The Rank and the Index of a Nilsoliton. The following proposition and its following corollary help us to compute rank of a nilsoliton metric Lie algebra.

Proposition 1 (see Proposition 4.7 in [9]). Let $\eta$ be a nonabelian Lie algebra that admits a simple derivation $D$. Let $B=\{X i\}_{i=1}^{n}$ be an eigenvector basis with index set $\Lambda$, and let $Y$ be the root matrix associated to $\Lambda$. Then, the rank of the nilsoliton metric Lie algebra $\eta$ equals to the nullity of the root matrix $Y$.

Corollary 1. Let $\eta$ be a nonabelian $n$-dimensional Lie algebra that admits a simple derivation. Let $B$ be an eigenvector basis with index set $\Lambda$, and let $Y$ be the root matrix associated to $\Lambda$. Then,

$$
\operatorname{rank}(\eta)=n+\operatorname{nullity}\left(Y Y^{t}\right)-|\Lambda|
$$

Proof. Suppose that $\eta$ is an $n$-dimensional nonabelian Lie algebra, admitting a simple derivation. Let $B$ be an eigenvector basis with index set $\Lambda$ where $|\Lambda|=m$, and let $Y$ be the root matrix associated to $\Lambda$. By Proposition 1, the root matrix $Y$ is an $m \times n$ matrix, whose nullity is $\operatorname{rank}(\eta)$. Therefore, from rank-nullity theorem, $\operatorname{rank}(\eta)=$ $n-\operatorname{Rank}(Y)$. We also know that $\operatorname{rank}(Y)=\operatorname{rank}\left(Y Y^{T}\right)$; therefore, we have

$$
\operatorname{rank}(\eta)=n-\operatorname{Rank}\left(Y Y^{T}\right)
$$

On the other hand, $Y Y^{T}$ is an $m \times m$ matrix. Therefore, $\operatorname{Rank}\left(Y Y^{T}\right)=m-\operatorname{Nullity}\left(Y Y^{T}\right)$. Then, we have

$$
\begin{aligned}
\operatorname{rank}(\eta) & =n-\left(m-\operatorname{Nullity}\left(Y Y^{T}\right)\right) \\
& =n+\operatorname{Nullity}\left(Y Y^{T}\right)-|\Lambda|
\end{aligned}
$$

Definition 4. Let $X \in \eta$, $a d_{X}$, denote the adjoint representation and $\eta^{*}$ denote the dual of the Lie algebra $\eta$. Then, the skew symmetric bilinear form $\Psi_{f}$, where $f \in \eta^{*}$, is defined by

$$
\begin{aligned}
\Psi_{f}: \eta \times \eta & \longrightarrow \mathbb{R} \\
(X, Y) & \longrightarrow \Psi_{f}(X, Y) \\
& =f([X, Y]) .
\end{aligned}
$$

The index of a Lie algebra $\eta$ is the integer $\inf \left\{\operatorname{dim} \eta_{f}: f \in \eta^{*}\right\} \quad$ where $\eta_{f}=\operatorname{ker}\left(\Psi_{f}\right)$ defined by $\eta_{f}=\{X \in \eta: f([X, Y])=0, \forall y \in \eta\}$.

Proposition 2 (see Proposition 4 in [10]). The index of an $n$-dimensional Lie algebra $\eta$ is the integer,

$$
\operatorname{index}_{\eta}=n-\operatorname{Rank}_{R(\eta)}\left(\left[X_{i}, X_{j}\right]\right)_{1 \leq i, j \leq n}
$$

where $R(\eta)$ is the quotient field of symmetric algebra $S(\eta)$.

Remark 3. above proposition tells us that the index of a Lie algebra is the nullity of the matrix $E_{\eta}=\left(\left[X_{i}, X_{j}\right]\right)$.

Now, we present an example regarding to this notion with the use of the index set $\Lambda$.

Example 2. Suppose that $\Lambda=\{(1,2,3),(1,3,4),(2,3,5)\}$ is the index set of an algebra $\eta$ of dimension 6 . Its corresponding Gram matrix is

$$
\left(\begin{array}{lll}
3 & 0 & 0 \\
0 & 3 & 1 \\
0 & 1 & 3
\end{array}\right) .
$$

Since there is no -1 entry, $\eta$ is a Lie algebra. The matrix $E_{\eta}$ is as follows:

$$
\left(\begin{array}{cccccc}
0 & X_{3} & X_{4} & 0 & 0 & 0 \\
-X_{3} & 0 & X_{5} & 0 & 0 & 0 \\
-X_{4} & -X_{5} & 0 & 0 & 0 & 0 \\
0 & 0 & 0 & 0 & 0 & 0 \\
0 & 0 & 0 & 0 & 0 & 0
\end{array}\right) .
$$

Its rank is 2 ; therefore, index $\log _{\eta}=4$.

Proposition 3. For an $n$-dimensional nilsoliton represented by an index set $\Lambda$ with a Nikolayevsky basis, the matrix $E_{\eta}$ has the following properties:

(1) The matrix is an $n \times n$ matrix with zero diagonal entries

(2) The rank of $E_{\eta}$ is even

(3) The $n^{\text {th }}$ row and column of $E_{\eta}$ are zero matrices

(4) If $|\Lambda|=K$, then $E_{\eta}$ has $2 K$ nonzero entries

Proof. Suppose that the Nikolayevsky basis is represented by $B=\left\{X_{\alpha}\right\}_{1 \leq \alpha \leq n}$. By the definition of $E_{\eta}, E_{\eta}(i, j)=\left[X_{i}, X_{j}\right]$, then

$$
\left(E_{\eta}\right)_{i j}=\left[X_{i}, X_{j}\right]=-\left[X_{j}, X_{i}\right]=-\left(E_{\eta}\right)_{j i} .
$$

Therefore, $E_{\eta}$ is a skew symmetric matrix, which implies the first and second properties. On the other hand, since $\eta$ is 
nilpotent Lie algebra with Nikolayevsky basis, then $\Lambda$ consists of the elements of form $(i, j, k)$ such that $i<j<k$. Therefore, $n$ cannot appear in the first or the second component of the triples in $\Lambda$. Thus, $\left[X_{i}, X_{n}\right]=\left[X_{n}, X_{i}\right]=0$ for all $i \in\{1,2, \ldots, n\}$, which implies that $\left(E_{\eta}\right)_{i n}=\left(E_{\eta}\right)_{n i}=0$, i.e., the last row and the last column are zero matrices.

The entries of $E_{\eta}$ are defined by the index set $\Lambda$. If $|\Lambda|=K$, then there exists $K$ non-zero entries on the upper triangular part of $E_{\eta}$. Since the matrix is skew symmetric, then there exists other $K$ nonzero entries in the lower triangular part of the matrix. So, in total, there exists $2 K$ nonzero entries in $E_{\eta}$.

Corollary 2. Suppose that $\eta$ is an $n$-dimensional nilsoliton. Then, index $(\eta)$ is even, if $n$ is even, and index is odd if $n$ is odd.

Proof. The index of the nilsoliton is $\operatorname{index}(\eta)=\operatorname{Nullity}\left(E_{\eta}\right)=n-\operatorname{rank}\left(E_{\eta}\right)$. Since $E_{\eta}$ is a skew symmetric matrix, its rank is always even, which finishes the proof.

\section{Conclusion}

In this paper, we prove theorems for the computations of structural elements of an $n$-dimensional nilsoliton $\eta$. We prove theorems regarding to the Jacobi identity or identities that have to be satisfied, rank, and index of the nilsoliton. In the future, we plan to use these theorems to create a computer algorithm for the classifications of nilsolitons for a given dimension. The theorems appearing in this study will allow us to pare down the number of cases to consider in our procedure.

\section{Data Availability}

No data were used in this study.

\section{Conflicts of Interest}

The author declares no conflicts of interest.

\section{References}

[1] J. Lauret and E. Solvmanifolds, "In Einstein solvmanifolds and nilsolitons," New Developments in Lie Theory and Geometry, vol. 23, 2009.

[2] J. Lauret and C. Will, "Einstein solvmanifolds: existence and non-existence questions," Mathematische Annalen, vol. 350, no. 1, pp. 199-225, 2011.

[3] De Graaf and A. Willem, Lie Algebras, Theory and Algorithms, Elsevier, London, UK, 2000.

[4] H. Kadioglu and T. L. Payne, "Computational methods for nilsoliton metric Lie algebras I," Journal of Symbolic Computation, vol. 50, pp. 350-373, 2013.

[5] H. Kadioglu, "Classification of ordered type soliton metric Lie algebras by a computational approach," Abstract and Applied Analysis, vol. 2013, 2013.

[6] H. Kadioglu, "A computational procedure on higher-dimensional nilsolitons," Mathematical Methods in the Applied Sciences, vol. 42, no. 16, pp. 5390-5397, 2019.
[7] Y. Nikolayevsky, "Einstein solvmanifolds with a simple Einstein derivation," Geometriae Dedicata, vol. 135, no. 1, pp. 87-102, 2008.

[8] T. L. Payne, "The existence of soliton metrics for nilpotent Lie groups," Geometriae Dedicata, vol. 145, no. 1, pp. 71-88, 2010.

[9] T. L. Payne, "Applications of index sets and Nikolayevsky derivations to positive rank nilpotent Lie algebras," Journal of Lie Theory, vol. 24, no. 1, 2014.

[10] H. Adimi and A. Makhlouf, "Computing the index of Lie algebras," Proceedings of the Estonian Academy of Sciences, vol. 59, no. 4, pp. 265-271, 2010. 